\title{
Identification of genes and genetic networks associated with BAG3-dependent cell proliferation and cell survival in human cervical cancer HeLa cells
}

\author{
YUKIHIRO FURUSAWA ${ }^{1}$, TATSUYA YUNOKI ${ }^{2}$, TETSUSHI HIRANO ${ }^{3}$, SATSUKI MINAGAWA ${ }^{3}$, \\ HIRONORI IZUMI $^{4}$, HISASHI MORI $^{4}$, ATSUSHI HAYASHI ${ }^{2}$ and YOSHIAKI TABUCHI $^{3}$ \\ ${ }^{1}$ Department of Liberal Arts and Sciences, Toyama Prefectural University, Toyama 939-0398; \\ ${ }^{2}$ Department of Ophthalmology, Graduate School of Medicine and Pharmaceutical Sciences; \\ ${ }^{3}$ Division of Molecular Genetics Research, Life Science Research Center; ${ }^{4}$ Department of Molecular Neuroscience, \\ Graduate School of Medicine and Pharmaceutical Sciences, University of Toyama, Toyama 930-0194, Japan
}

Received April 20, 2018; Accepted July 17, 2018

DOI: $10.3892 / \mathrm{mmr} .2018 .9383$

\begin{abstract}
Bcl-2-associated athanogene (BAG) 3, is a member of the BAG protein family and a known co-chaperone of heat shock protein (HSP) 70. BAG3 serves a role in regulating a variety of cellular functions, including cell growth, proliferation and cell death including apoptosis. BAG3 is a stress-inducible protein, however the constitutive expression level of BAG3 is increased in cancer cells compared with healthy cells. Recent proteomics technology combined with bioinformatics has revealed that BAG3 participates in an interactome with
\end{abstract}

Correspondence to: Professor Yoshiaki Tabuchi, Division of Molecular Genetics Research, Life Science Research Center, University of Toyama, 2630 Sugitani, Toyama 930-0194, Japan

E-mail: ytabu@cts.u-toyama.ac.jp

Dr Yukihiro Furusawa, Department of Liberal Arts and Sciences, Toyama Prefectural University, 5180 Kurokawa, Toyama 939-0398, Japan

E-mail: furusawa@pu-toyama.ac.jp

Abbreviations: BAG, BCL2-associated athanogene; BMP2, bone morphogenetic protein 2; CCND1, cyclin D1; Cas9, CRISPR associated protein 9; CRISPR, clustered regularly interspaced short palindromic repeats; EPCAM, epithelial cell adhesion molecule; ERBB4, erb-b2 receptor tyrosine kinase 4; EGR1, early growth response 1; F2R, coagulation factor II thrombin receptor; IPA, Ingenuity Pathways Analysis; KLF4, Kruppel like factor 4; NOV, nephroblastoma overexpressed; PCA, principal component analyses; PDGFC, platelet derived growth factor C; PPARG, peroxisome proliferator activated receptor $\gamma$; PTHLH, parathyroid hormone like hormone; QUICK, quantitative immunoprecipitation combined with knockdown; RUNX2, runt-related transcription factor 2; TIMP3, TIMP metallopeptidase inhibitor 3

Key words: BCL2-associated athanogene 3, proliferation, cell survival, gene expression, microarray, human cervical cancer $\mathrm{HeLa}$ cell a number of proteins other than its typical partner HSP70. The functional types represented in the interactome included nucleic acid binding proteins and transcription factors, as well as chaperones, which indicated that overexpression of BAG3 may contribute to proliferation and cell survival through the alteration of gene transcription. While an increasing number of studies have addressed the function of BAG3 as a co-chaperone protein, BAG3-dependent alteration of gene transcription has not been studied extensively. The present study established two BAG3 knockout human cervical cancer HeLa cell clones and addressed the role of BAG3 in cell proliferation and survival through gene transcription, using DNA microarray-based transcriptome analysis and bioinformatics. The present study also identified two genetic networks associated with 'cellular growth and proliferation' and 'cell death and survival', which are dysregulated in the absence of BAG3, and may therefore be linked to BAG3 overexpression in cancer. These findings provide a molecular basis for understanding of BAG3-dependent cell proliferation and survival from the aspect of alteration of gene expression.

\section{Introduction}

Bcl-2 associated athanogene (BAG) 3 is a member of the human BAG family of co-chaperone proteins (1). The BAG domain of BAG3 protein binds to heat shock protein (HSP) 70, a major chaperone protein involved in anti-apoptosis through recovery of unfolded proteins as well as interference with pro-apoptotic cytochrome c release from mitochondria (2). BAG3 is also known to bind to phospholipase $\mathrm{C}-\gamma$ and $\mathrm{Bcl}-2$, which synergistically inhibit cell death (3). In addition, BAG3 was reported to interact with dual-specificity phosphatase 6 , which is involved in extracellular signal-regulated kinase de-phosphorylation, resulting in the induction of cell proliferation (4). BAG3 possesses an N-terminal WW domain and a C-terminal PxxP domain that interact with partner proteins other than HSP70, resulting in modulation of various biological processes such as anti-apoptosis, proliferation, cell adhesion, metastasis, invasion, and autophagy $(3,5-7)$. 
Under physiological conditions, constitutive expression levels of BAG3 are low in normal cells other than muscle cells. BAG3 expression is induced under stress conditions such as heavy metals, heat, oxidative stress, ultrasound, and starvation (8-13). The expression of BAG3 is reported to be regulated partially by the activation of heat shock transcription factor 1 as in the cases of HSPs (14). In addition, BAG3 and some HSP family proteins are controlled by hypoxia-induced factor 1 , which is highly expressed under hypoxic conditions, such as in tumor microenvironments (15). However, many studies have revealed that BAG3 expression is also elevated under normoxic conditions in numerous tumor cells including breast cancer, prostate cancer, ovarian cancer, colorectal cancer, melanoma, and osteosarcoma $(3,6,16-19)$. This is probably because BAG3 contributes to cell proliferation as well as cell survival through interaction with anti-apoptotic proteins such as Bcl-2, and myeloid leukemia cell differentiation protein 1 that are overexpressed in cancer (20). Indeed, overexpression of BAG3 correlates with dismal prognosis in melanoma and several carcinomas $(6,16,18)$.

An impressive recent study revealed functional categories of BAG3 partner proteins by using novel comprehensive proteome analysis, called quantitative immunoprecipitation combined with knockdown (QUICK) (21). Protein analysis through evolutionary relationships classification of the data obtained from QUICK demonstrated that the BAG3 interactome includes transcription factors, indicating that BAG3-dependent cell proliferation and survival may be mediated by gene transcription, at least in part. Despite accumulating data on the functions of BAG3 and its protein-protein interactions, neither the transcripts associated with BAG3 overexpression in cancer cells, nor their functions, are fully elucidated. Here, we performed DNA microarray-based comprehensive transcriptome analysis and bioinformatics on two BAG3 knockout (KO) HeLa cell clones established using the clustered regularly interspaced short palindromic repeats (CRISPR)-Cas9 (CRISPR associated protein 9) genome editing system. Finally, we identified genetic networks of transcripts associated with proliferation and cell survival, which may be dependent on BAG3 expression.

\section{Materials and methods}

Cell culture. Human cervical cancer HeLa cells were newly obtained from the Human Science Research Resources Bank, Japan Health Sciences Foundation (Tokyo, Japan) for this experiment. Cells were cultured in E-MEM (Wako Pure Chemical Industries, Ltd., Osaka, Japan) supplemented $10 \%$ fetal bovine serum (Equitech-Bio, Inc., Kerrville, TX, USA) and $1 \%$ penicillin/streptomycin (Nacali Tesque, Inc., Kyoto, Japan).

Establishment of BAG3 KO HeLa cell clones. In the present study, the BAG3 gene was deleted using the CRISPR-Cas9 genome editing system as described below. For the expression of Cas9 protein and guide RNA targeting the BAG3 gene, pX362 vector (Addgene, Cambridge, MA, USA) was used as previously described (22). After the digestion of pX362 with BbsI, the oligonucleotides 5'-caccGAGACTCCATCCTC TGCCAA-3' and 5'-aaacTTGGCAGAGGATGGAGTCTC-3' (upper and lower case letters are protospacer sequence and additional sequence to clone into the BbsI site, respectively) corresponding to the single guide RNA target sequence in exon 1 of BAG3 were annealed and subcloned into the BbsI site of pX362. The constructed plasmid was transfected into HeLa cells with Effectene Transfection Reagent (Qiagen $\mathrm{GmbH}$, Hilden, Germany) according to the manufacture's procedure. The DNA-transfected HeLa cells were selected by treatment with $1 \mu \mathrm{g} / \mathrm{ml}$ of puromycin (Thermo Fisher Scientific, Inc., Waltham, MA, USA) for $48 \mathrm{~h}$, followed by limited dilution to obtain the colonies. The grown colonies were picked up and expanded. After initial screening by immunoblotting with anti-BAG3 antibody, genomic DNA from the HeLa cell clones was prepared and used for polymerase chain reaction to examine the sequence of the DNA fragment containing the target site. The forward and reverse primer sequences were 5'-CCAGCCTGTGTTTCTCCACTT-3' and 5'-CTGTCTTTG CTGGGTGACCT-3', respectively.

SDS-PAGE and western blot analysis. Cells were dissolved in a lysis buffer $(150 \mathrm{mM} \mathrm{NaCl}, 1 \%$ Nonidet P-40 and $50 \mathrm{mM}$ Tris-HCl, $\mathrm{pH}$ 8.0) containing a protease inhibitor cocktail (Nacali Tesque, Inc.). SDS-PAGE and Western blotting were carried out as described elsewhere (12). Proteins were detected using the following primary antibodies: Anti-BAG3 rabbit monoclonal antibody (GeneTex Inc., Irvine, CA, USA) and anti-glyceraldehyde 3-phosphate dehydrogenase (GAPDH) mouse monoclonal antibody (as a loading reference; Proteintech, Rosemont, IL, USA). Secondary fluorescent-conjugated anti-mouse and anti-rabbit IgGs (LI-COR Bioscience, Lincoln, NE, USA) were also used. Protein expression levels and images were acquired using an Odyssey Infrared Imager (LI-COR Biosciences).

Cell counting assay. Ten thousand cells were seeded in 24-well culture plates for cell counting. After trypsinization, the trypan blue dye exclusion test was performed, by mixing cell suspension with an equal amount of phosphate-buffered saline containing $0.4 \%$ trypan blue. The number of cells excluding the dye was counted by using an $\mathrm{EVE}^{\mathrm{TM}}$ automatic cell counter (NanoEnTek, Inc., Seoul, Korea).

Cell cycle analysis. Fifty thousand cells were cultured in $60 \mathrm{~mm}$ culture dishes two days before cell cycle analysis. For flow cytometry, cells were fixed with $70 \%$ ice cold ethanol for at least $1 \mathrm{~h}$, and subsequently treated with $0.25 \mathrm{mg} / \mathrm{ml}$ RNase A (Nacali Tesque, Inc.) and SYTOX AADvanced (Thermo Fisher Scientific, Inc.) as described (23). The samples were finally analyzed by flow cytometry using a Novocyte flow cytometer (Novocyte, San Diego, CA, USA). A total of 10,000 cells per sample were analyzed in each experiment. Distribution of cells in each cell cycle phase was analyzed based on the Watson model (24).

RNA isolation. Total RNA was extracted from cells using a NucleoSpin ${ }^{\circledR}$ RNA isolation kit (Macherey-Nagel GmbH \& Co., Düren, Germany) and treated with DNase I for 20 min at room temperature to remove residual genomic DNA. The quality of the RNA was analyzed using a Bioanalyzer 2100 and a RNA6000 Nano LabChip kit (Agilent Technologies, 

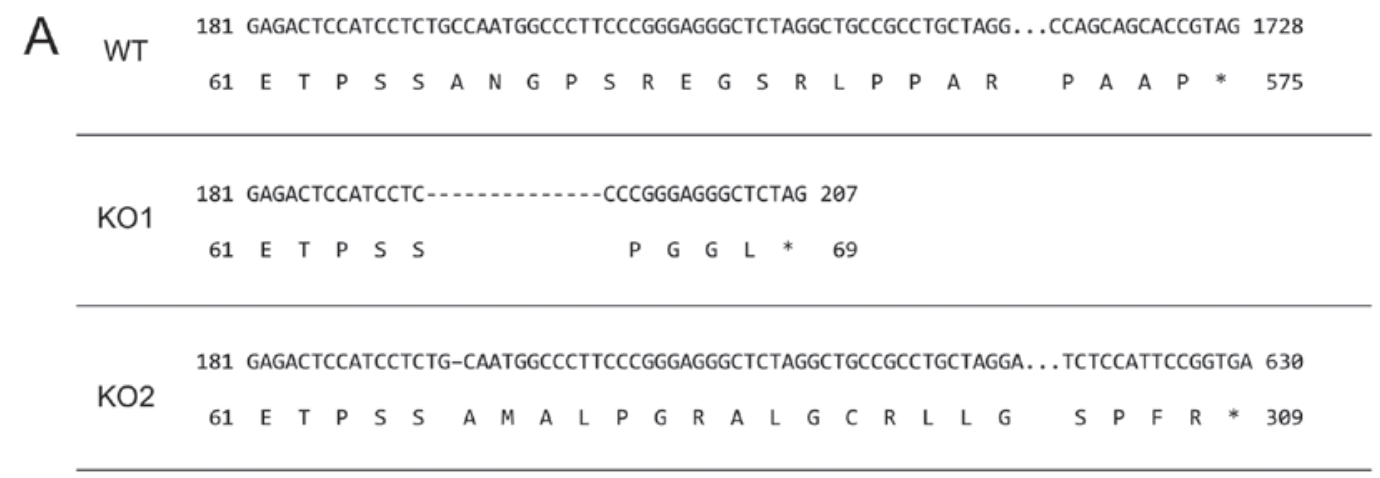

B

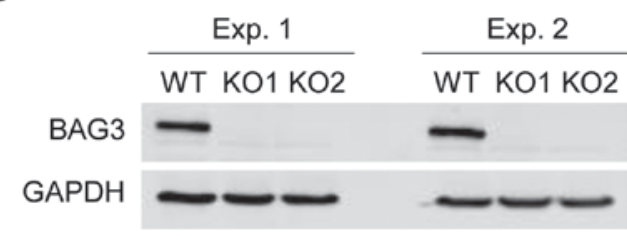

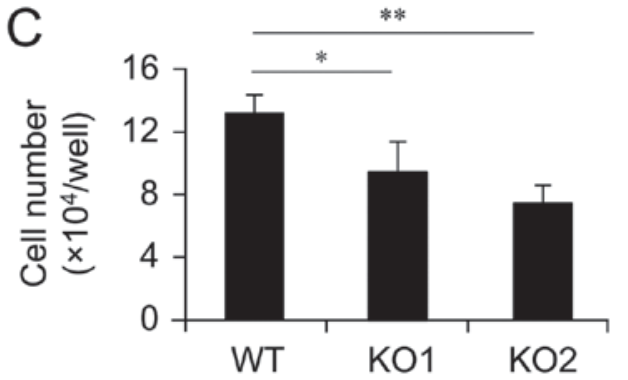

Figure 1. Establishment of BAG3 KO HeLa cell clones. (A) Genomic DNA sequence of a part of the human BAG3 exon 1 and deduced amino acid sequence of human BAG3 in BAG3 WT HeLa cells and BAG3 KO HeLa cell clones, KO1 and KO2. (B) Western blot analysis of BAG3 protein from WT cells and two established BAG3 KO clones. An anti-BAG3 antibody was used as primary antibody. The experiment was performed twice. (C) The cell number after plating. Cells were counted 7 days after plating in 24 -well plates. Data are presented as the mean \pm standard deviation $(\mathrm{n}=4)$. ${ }^{*} \mathrm{P}<0.05$, ${ }^{* *} \mathrm{P}<0.01$. KO, knockout; $\mathrm{WT}$, wild-type; BAG3, Bcl-2-associated athanogene; GAPDH, glyceraldehyde 3-phosphate dehydrogenase.

Inc., Santa Clara, CA, USA). RNA samples with RNA integrity number values above 9.5 were considered acceptable.

Gene expression analysis. Microarray and computational gene expression analyses were performed using a GeneChip ${ }^{\circledR}$ system with a Human Genome U133-plus 2.0 array (Affymetrix, Inc., Santa Clara, CA, USA), which was spotted with $\sim 55,000$ probe sets, as previously described. Samples for array hybridization were prepared as described in the Affymetrix GeneChip ${ }^{\circledR}$ Expression Technical Manual. The scanned arrays were analyzed using the GeneChip Analysis Suite Software (Affymetrix, Inc.). The microarray data were deposited in the Gene Expression Omnibus: http://www.ncbi. nlm.nih.gov/geo/query/acc.cgi?acc=GSE103475.

For global normalization, microarray signals were processed using a standard MAS5.0 algorithm. The obtained hybridization intensity data and qualities were checked using the GeneSpring ${ }^{\circledR}$ software (Agilent Technologies, Inc.). Observed signals were normalized and genes that had no significant signals were ignored to reduce noise. In addition, probe sets targeting specific RefSeq transcripts, based on the RefDIC database, were extracted (25). Principal component analyses (PCA) were performed using R v3.2.1. Venn diagrams and hierarchical clustering from the obtained normalized intensity data were produced using GeneSpring ${ }^{\circledR}$ software. Ward's linkage and squared Euclidean distance were utilized in hierarchical clustering.

In order to examine the molecular functions of differentially expressed genes and gene networks, data were analyzed using Ingenuity Pathways Analysis (IPA) tools (Ingenuity Systems, Mountain View, CA, USA), a web-delivered application that enables the identification, visualization, and exploration of molecular interaction networks in gene expression data. The top five molecular functions were identified and the gene networks containing the molecules were visualized to provide information about interactions involving genes that were up- or downregulated by BAG3 deletion.

Statistical analysis. Data are presented as the mean \pm standard deviation (SD). The statistical significance of differences between data sets was analyzed using one-way analysis of variance (ANOVA) with post-hoc Tukey HSD tests ( $\mathrm{R}$ v3.2.1). $\mathrm{P}<0.05$ was considered to indicate a statistically significant difference. In microarray analysis, raw $\mathrm{P}$-values were adjusted by calculating false discovery rate using the Benjamini-Hochberg method (GeneSpring ${ }^{\circledR}$ software; Agilent Technologies, Inc.).

\section{Results}

Establishment and characterization of BAG3 KO HeLa cell clones. To confirm the role of BAG3 in cell proliferation and survival in HeLa cells, we constructed BAG3 KO HeLa cell clones using a CRISPR-Cas9 genome editing system. Among isolated clones, two clones, designated as $\mathrm{KO} 1$ and $\mathrm{KO} 2$, were selected as BAG3 KO candidates. This selection was based on the result of initial screening by western blotting using anti-BAG3 antibody, since it is possible that translation can be initiated from an in-frame ATG in nonsense mutation near the $5^{\prime}$ region of an open reading frame (26). After the initial screening, we confirmed the sequence of exon 1 of the BAG3 gene and found that two clones contained a 14 or 1 bp deletion 
A
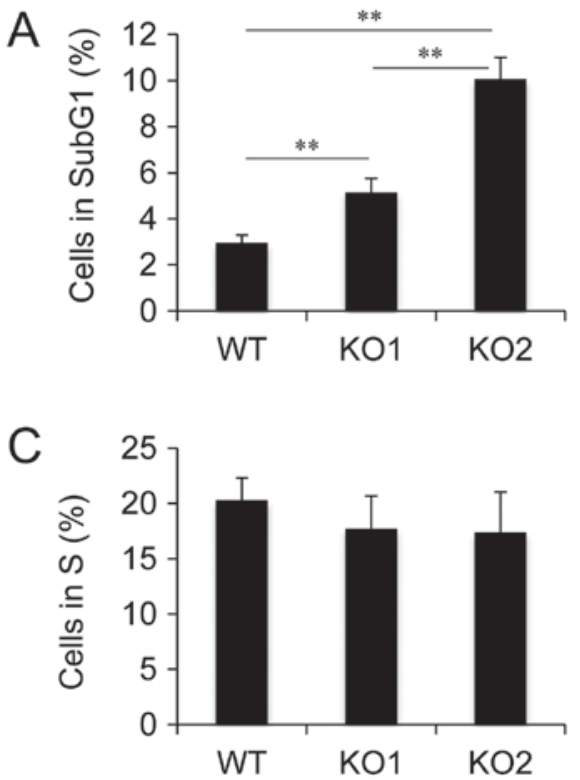

$\mathrm{B}$
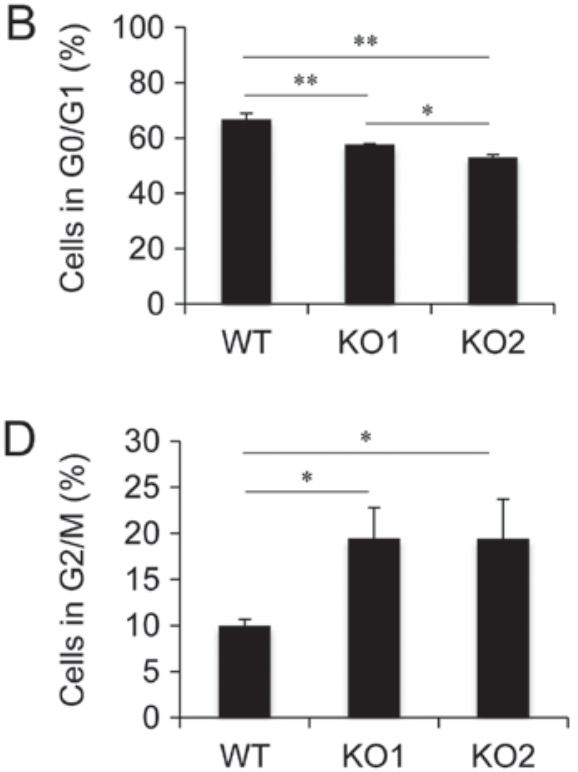

Figure 2. Cell cycle analysis of BAG3 KO HeLa cell clones. Distribution of cells in the (A) SubG1, (B) G0/G1, (C) S and (D) G2/M phases in BAG3 WT Hela cells and BAG3 KO HeLa cell clones. Data are presented as the mean \pm standard deviation $(\mathrm{n}=3)$. ${ }^{*} \mathrm{P}<0.05,{ }^{* *} \mathrm{P}<0.01$. KO, knockout; WT, wild-type; BAG3, Bcl-2-associated athanogene.

in exon 1 of both alleles, respectively, resulting in a frame-shift and premature termination of BAG3 translation (Fig. 1A). We confirmed that BAG3 protein expression was diminished as a result of the deletions in the BAG3 gene by repeated western blot analysis (Fig. 1B). The two selected BAG3 KO clones and the wild-type (WT) control cells were cultured for 7 days to assess whether BAG3 deletion affected the number of viable cells in culture conditions. As a result of BAG3 deletion, the number of viable cells was significantly decreased in both clones (Fig. 1C). This result is consistent with involvement of BAG3 with cell proliferation and/or cell survival even under culture conditions without exogenous cytotoxic stimulation (e.g., heat or oxidative stress).

Cell cycle distribution in BAG3 KO HeLa cell clones. Established BAG3 KO HeLa cell clones showed decreased numbers of viable cells, indicating that BAG3 deletion led to cell cycle delay and/or cell death. To examine the effect of BAG3 on cell cycle progression and cell survival during cell culture, we performed cell cycle analysis using flow cytometry. Cell cycle analysis revealed that the populations of cells in Sub G1 and $\mathrm{G} 2 / \mathrm{M}$ phase were increased in both BAG3 KO clones (Fig. 2), indicating that BAG3 is involved in both cell cycle progression and anti-apoptosis under normal culture conditions in $\mathrm{HeLa}$ cells.

Global gene expression analysis in BAG3 KO HeLa cell clones. In order to analyze gene expression associated with BAG3, we performed microarray analysis in WT and two BAG3 KO HeLa cell clones. After normalization of obtained intensities using the MAS5.0 algorithm, we performed PCA on gene expression data. This revealed that the gene expression patterns in WT cells were markedly distinct from those in BAG3 KO cells (Fig. 3A). However, the gene expression pattern in clone $\mathrm{KO} 1$ was also distinct from that in $\mathrm{KO}$ 2, which we assumed was due to normal/stochastic heterogeneity between isolated clones.
Therefore, we attempted to identify common up- or downregulated genes between two established clones to narrow down the potential BAG3-target genes. Of the 54,675 probe sets analyzed, 28,719 reliable probe sets were extracted using RefDIC database, since the probes on this type of array include unreliable probes that were designed based on a classical database. Among them, 5,436 probe sets were defined as statistically significant based on one-way ANOVA with post-hoc Tukey HSD and Benjamini-Hochberg procedure. Furthermore, we identified 1,274 probe sets that were differentially expressed by a factor of 2.0 or greater in either WT cells or BAG3 KO clones. Among them, 102 and 284 probe sets were commonly up- or downregulated in BAG3 KO clones, respectively (Fig. 3B).

Computational analysis of genes responsive to BAG3 deletion and gene network analysis. Venn diagrams of differentially expressed probes showed that 102 and 284 genes were up- and downregulated by BAG3 deletion, respectively. In the present study, we further performed a bioinformatics analysis to identify the molecular/cellular functions, and the genetic networks of differentially expressed genes, in order to elucidate the mechanisms underlying cell death and cell cycle delay in response to BAG3 deletion. From the analysis of molecular and cellular function based on IPA knowledge base, we found that 'cell death and cell survival' and 'cellular growth and proliferation' were among the top five functions for up- and downregulated genes, respectively (Table I).

In order to elucidate the interactions between the up- or downregulated genes, we performed a gene network analysis. The analysis identified an upregulated gene network (Network up) containing 12 genes, including cyclin D1 (CCND1), matrix metalloproteinase 2 (MMP2), platelet derived growth factor $\mathrm{C}$ (PDGFC), runt-related transcription factor 2 (RUNX2), peroxisome proliferator-activated receptor $\gamma$ (PPARG) and coagulation factor II thrombin receptor (F2R) (Fig. 4A and Table II). The downregulated gene network (Network down) contained 
Table I. Top five molecular and cellular functions in up- and downregulated genes.

A, Upregulated

Molecular and cellular function

P-value

Numbers of molecules

Cell morphology

1.45E-02-3.31E-08

71

Cellular development

$1.45 \mathrm{E}-02-4.67 \mathrm{E}-06$

70

Cellular growth and proliferation

1.29E-02-4.67E-06

55

Cellular assembly and organization

$1.23 \mathrm{E}-02-4.81 \mathrm{E}-06$

44

Cellular function and maintenance

1.29E-02-4.81E-06

47

B, Downregulated

Molecular and cellular function

P-value

Numbers of molecules

Cell-to-cell signaling and interaction

$\begin{array}{ll}1.25 \mathrm{E}-02-2.12 \mathrm{E}-07 & 22 \\ 1.16 \mathrm{E}-02-9.03 \mathrm{E}-07 & 27 \\ 1.25 \mathrm{E}-02-4.49 \mathrm{E}-06 & 30 \\ 1.25 \mathrm{E}-02-2.97 \mathrm{E}-05 & 21 \\ 1.21 \mathrm{E}-02-4.05 \mathrm{E}-05 & 31\end{array}$

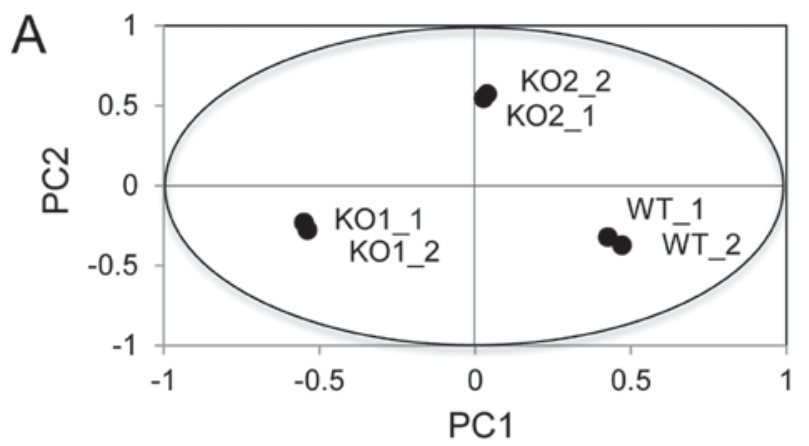

Cellular movement

Cell death and survival

Cell morphology

Cellular development

\section{B Upregulated ( $\geq 2.0$ fold)}

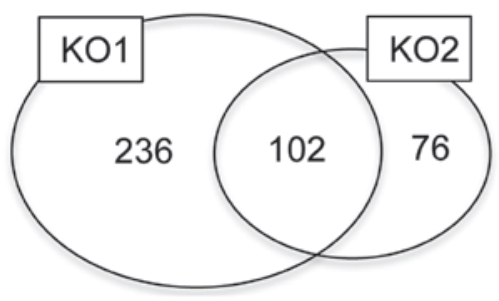

Downregulated ( $\geq 2.0$ fold)

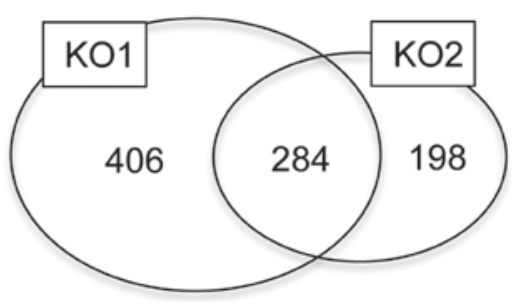

Figure 3. Microarray-based global gene expression analysis of BAG3 KO HeLa cell clones. (A) PCA on the comprehensive gene expression analysis data. PCA was performed using Gene Spring Software. (B) Venn-diagrams of 2.0-fold up- or downregulated genes in the two established BAG3 KO clones. PCA, principal component analysis; KO, knockout; BAG3, Bcl-2-associated athanogene.

15 genes, including TIMP metallopeptidase inhibitor 3 (TIMP3), Krupper like factor 4 (KLF4), epithelial cell adhesion molecule (EPCAM), erb-b2 receptor tyrosine kinase 4 (ERBB4), and bone morphogenetic protein 2 (BMP2) (Fig. 4B and Table II). Among them, BAG3 has already been reported to interact with MMP2 and PDGFC $(6,21)$, but not with other genes. In addition, the BioGRID database, a depository of interaction datasets including the results of recent interactome analyses, also did not report interaction between BAG3 and the genes in the networks we identified, other than MMP2 and PDGFC, suggesting that the expression changes of most transcripts identified here may have been independent of BAG3 interaction with proteins coded by these genes.

\section{Discussion}

The DNA microarray has been a standard technology for elucidating genome-wide gene expression signatures in life 
Table II. Genes in two identified genetic networks.

A, Upregulated (network up)

\begin{tabular}{llll}
\hline & \multicolumn{2}{l}{ Fold change (vs. WT) } & \\
\cline { 2 - 3 } Gene symbol & KO1 & KO2 & \\
\hline MMP2 & 3.41 & 5.28 & Matrix metallopeptidase 2 \\
SCG5 & 7.03 & 5.12 & Secretogranin V \\
F2R & 2.93 & 3.29 & Coagulation factor II thrombin receptor \\
NOV & 2.47 & 6.06 & Nephroblastoma overexpressed \\
PTHLH & 3.85 & 2.68 & Parathyroid hormone like hormone \\
IL11 & 3.00 & 3.76 & Interleukin 11 \\
PPARG & 2.97 & 3.02 & Peroxisome proliferator activated receptor $\gamma$ \\
CCND1 & 5.87 & 5.00 & Cyclin D1 \\
MEF2C & 2.55 & Myocyte enhancer factor 2C \\
PDGFC & 2.20 & 2.58 & Platelet derived growth factor C \\
GNG2 & 4.09 & 3.03 & G protein subunit $\gamma$ 2 \\
RUNX2 & 3.48 & 6.96 & Runt related transcription factor 2 \\
\hline
\end{tabular}

B, Downregulated (network down)

\begin{tabular}{llll}
\hline & \multicolumn{2}{l}{ Fold change (vs. WT) } & \\
\cline { 2 - 3 } Gene symbol & KO1 & KO2 & \\
\hline TIMP3 & 0.49 & 0.28 & TIMP metallopeptidase inhibitor 3 \\
EGR1 & 0.11 & 0.32 & Early growth response 1 \\
EPCAM & 0.07 & 0.16 & Epithelial cell adhesion molecule \\
CRABP2 & 0.09 & 0.27 & Cellular retinoic acid binding protein 2 \\
HPGD & 0.10 & 0.26 & 15-hydroxyprostaglandin dehydrogenase \\
AREG & 0.22 & 0.43 & Amphiregulin \\
BMP2 & 0.18 & 0.45 & Bone morphogenetic protein 2 \\
NR4A3 & 0.15 & 0.35 & Nuclear receptor subfamily 4 group A member 3 \\
UCP2 & 0.27 & 0.32 & Uncoupling protein 2 \\
DCN & 0.30 & 0.20 & Decorin \\
CCNE2 & 0.46 & Cyclin E2 \\
ERBB4 & 0.49 & 0.26 & Erb-b2 receptor tyrosine kinase 4 \\
CCL2 & 0.18 & 0.16 & C-C motif chemokine ligand 2 \\
KLF4 & 0.24 & 0.49 & Kruppel like factor 4 \\
HEY1 & 0.44 & 0.45 & Hes related family bHLH transcription factor with YRPW motif 1 \\
\hline
\end{tabular}

WT, wild-type; KO, knockout.

science research fields. In this study, we addressed the role of BAG3 in gene transcription by combining transcriptome and computational analysis in two stable BAG3 KO HeLa cell clones. Currently, more than 400 BAG3 partner proteins are listed in public and commercial databases. Among them, we identified MMP2 and PDGFC as transcriptionally upregulated by BAG3 deletion. MMP2 is known to contribute to tumor cell apoptosis, probably through the degradation of poly (ADP-ribose) polymerase, which repairs DNA single-strand breaks $(27,28)$. In contrast, PDGFC shows anti-apoptotic effect and promotes cell proliferation $(29,30)$. These genes seem to compete with each other at the level of transcription and probably also at the post-translational level by interacting with BAG3. Interestingly, only these two genes were identified as BAG3-related genes in our gene networks. It is possible that the upregulation of MMP2 and PDGFC transcripts resulted from destabilization of MMP2 and PDGFC (negative feedback through protein degradation) in the absence of BAG3. Also of note, we found that the transcription factors RUNX2 and PPARG, not previously reported to interact with BAG3, were markedly upregulated by BAG3 deletion in two BAG3 KO clones established in this study. RUNX2 was reported to be involved in cell proliferation under normal conditions as well as cell survival under conditions of stress $(31,32)$. PPARG was overexpressed in cancer but its 



\section{Edge labels}
A, activation/deactivation
$\square$ Cytokine
- direct interaction
E, expression
G-protein-coupled receptor
LO, localization
P, phosphorylation/dephosphorylation
Growth factor
PD, protein-DNA binding
Kinase
PP, protein-protein binding
Nuclear receptor
$\mathbf{R B}$, regulation of binding
Others
$\mathrm{T}$, transcription
.$---- \quad$ indirect interaction
Upregulated
Downregulated

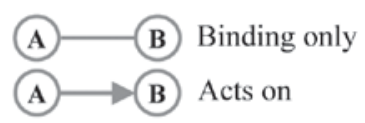

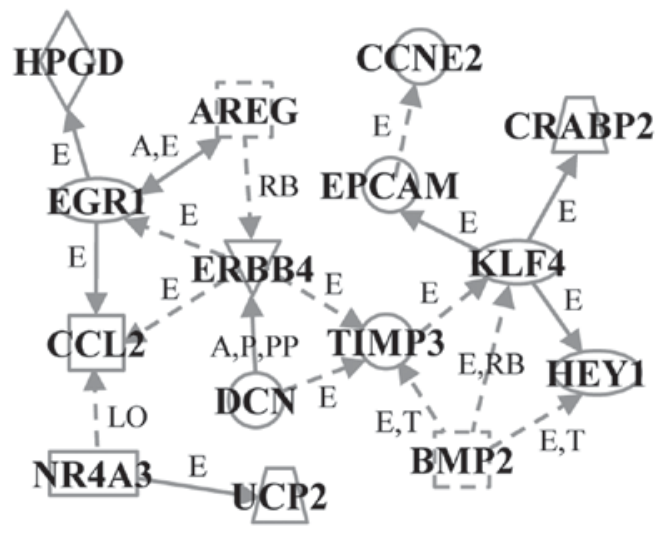

Figure 4. Genetic networks of genes up- or downregulated by BAG3 deletion in HeLa cell clones. (A) A genetic network of genes upregulated in BAG3 knockout HeLa cell clones (Network up). (B) A genetic network of genes dominantly expressed in wild-type HeLa cells. The network is represented graphically with nodes (genes) and edges (the biological associations between the nodes). BAG3, Bcl-2-associated athanogene.

excessive activation led to growth inhibition and apoptosis (33). Neither transcription factor has yet been reported to interact with BAG3, but both are known to enhance the expression of MMP2, indicating that BAG3 may be indirectly involved in RUNX2- and PPARG-dependent transcription of MMP2. BAG3 deletion also upregulated expression of CCND1, known to be a downstream target of RUNX2 and PPARG $(34,35)$. CCND1 is well known as a G1 cyclin, and degradation of this protein results in G1 arrest. However, overexpression of CCND1 perturbs normal replication and induces DNA damage (36), resulting in apoptotic cell death. Parathyroid hormone like hormone (PTHLH) is positive regulator of CCND1 transcription through RhoA/ROCK signaling (37). F2R is also known to promote CCND1 expression through the transcription factor c-Fos (38). Nephroblastoma overexpressed (NOV) inhibits cell proliferation when overexpressed in Ewing's sarcoma cells (39). It is conceivable that BAG3 indirectly suppresses transcription of the genes in the Network up to promote cell cycle progression and cell survival in HeLa cells.

In contrast to genes identified in the Network up, some genes were downregulated by BAG3 deletion. Among downregulated genes, ERBB4, TIMP3, KLF4, and BMP2 were located in the central region of the Network down. ERBB4 encodes
HER4, a tyrosine kinase receptor belonging to the epidermal grow th factor receptor family. ERBB4 is overexpressed in Ewing's sarcoma cells and activates the PI3K-Akt cascade, resulting in the promotion of cell growth and survival (40). In addition, ERBB4 is known to enhance the expression of downstream molecules such as early growth response 1 (EGR1) and TIMP3 (41). EGR1 is a transcription factor that plays a critical role in cell growth and survival $(42,43)$. TIMP3 acts as a tissue inhibitor of MMP2 (44). Thus, downregulation of ERBB4 and TIMP3 may lead to the activation of overexpressed MMP2 in BAG3 KO HeLa cells. TIMP3 also regulates the expression of KLF4. KLF4 is a transcription factor linked to tumor cell growth by a study showing that its downregulation inhibits the proliferation of cancer cells (45). Mutation of KLF4 leads to a decrease in the level of EPCAM (46), high expression of which in gastric cancer is linked to proliferation (47). BMP2 inhibits apoptosis through the activation of BMP receptor 2 (48). Furthermore, BMP2 was reported to upregulate expression of TIMP3 and KLF4 (49). The BMP2-TIMP3 and BMP2-KLF4 signaling axes may also contribute to proliferation and cell survival through BAG3-dependent transcription.

In this study, we identified two BAG3-dependent genetic networks associated with cellular growth and proliferation as 
well as cell death and survival. These findings will provide a molecular basis for understanding BAG3-dependent transcriptional regulation of genes in cancer cells. Further investigation is needed to identify the BAG3-binding, up-stream transcriptional regulators of the genes listed in the genetic networks we identified.

\section{Acknowledgements}

Not applicable.

\section{Funding}

The present study was supported in part by the JSPS KAKENHI (grant nos. 16K20309 and 17K01353).

\section{Availability of data and materials}

The datasets used and/or analyzed during the current study are available from the corresponding author on reasonable request.

\section{Authors' contributions}

YF and YT conceived the study, designed the experiments, wrote the manuscript and performed the experiments. TH and SM also performed the experiments. $\mathrm{HI}$ and HM provided the materials and performed genome editing. TY and AH provided materials for microarray analysis and were involved in data analysis.

\section{Ethics approval and consent to participate}

Not applicable.

\section{Patient consent for publication}

Not applicable.

\section{Competing interests}

The authors declare that they have no competing interests.

\section{References}

1. Behl C: Breaking BAG: The co-chaperone BAG3 in health and disease. Trends Pharmacol Sci 37: 672-688, 2016.

2. Rosati A, Ammirante M,Gentilella A, Basile A,Festa M,Pascale M, Marzullo L, Belisario MA, Tosco A, Franceschelli S, et al: Apoptosis inhibition in cancer cells: A novel molecular pathway that involves BAG3 protein. Int J Biochem Cell Biol 39: 1337-1342, 2017.

3. Kassis JN, Guancial EA, Doong H, Virador V and Kohn EC: CAIR-1/BAG-3 modulates cell adhesion and migration by downregulating activity of focal adhesion proteins. Exp Cell Res 312: 2962-2971, 2006.

4. Falco A, Festa M, Basile A, Rosati A, Pascale M, Florenzano F, Nori SL, Nicolin V, Di Benedetto M, Vecchione ML, et al: BAG3 controls angiogenesis through regulation of ERK phosphorylation. Oncogene 31: 5153-5161, 2012.

5. Shi H, Xu H, Li Z, Zhen Y, Wang B, Huo S, Xiao R and Xu Z: BAG3 regulates cell proliferation, migration, and invasion in human colorectal cancer. Tumour Biol 37: 5591-5597, 2016.

6. Suzuki M, Iwasaki M, Sugio A, Hishiya A, Tanaka R, Endo T, Takayama S and Saito T: BAG3 (BCL2-associated athanogene 3) interacts with MMP-2 to positively regulate invasion by ovarian carcinoma cells. Cancer Lett 303: 65-71, 2011.
7. Kathage B, GehlertS,Ulbricht A,Lüdecke L, Tapia VE, Orfanos Z, Wenzel D, Bloch W, Volkmer R, Fleischmann BK, et al: The cochaperone BAG3 coordinates protein synthesis and autophagy under mechanical strain through spatial regulation of mTORC1. Biochim Biophys Acta 1864: 62-75, 2017.

8. Bonelli P, Petrella A, Rosati A, Romano MF, Lerose R, Pagliuca MG, Amelio T, Festa M, Martire G, Venuta S, et al: BAG3 protein regulates stress-induced apoptosis in normal and neoplastic leukocytes. Leukemia 18: 358-360, 2004.

9. Tabuchi Y, Ando H, Takasaki I, Feril LB Jr, Zhao QL, Ogawa R, Kudo N, Tachibana K and Kondo T: Identification of genes responsive to low intensity pulsed ultrasound in a human leukemia cell line Molt-4. Cancer Lett 246: 149-156, 2007.

10. Jung SE, Kim YK, Youn DY, Lim MH, Ko JH, Ahn YS and Lee JH: Down-modulation of Bis sensitizes cell death in C6 glioma cells induced by oxygen-glucose deprivation. Brain Res 1349: 1-10, 2010.

11. Pagliuca MG, Lerose R, Cigliano S and Leone A: Regulation by heavy metals and temperature of the human BAG-3 gene, a modulator of Hsp70 activity. FEBS Lett 541: 11-15, 2003.

12. Yunoki T, Kariya A, Kondo T, Hayashi A and Tabuchi Y: The combination of silencing BAG3 and inhibition of the JNK pathway enhances hyperthermia sensitivity in human oral squamous cell carcinoma cells. Cancer Lett 335: 52-57, 2013.

13. Yunoki T, Tabuchi Y, Hayashi A and Kondo T: Network analysis of genes involved in the enhancement of hyperthermia sensitivity by the knockdown of BAG3 in human oral squamous cell carcinoma cells. Int J Mol Med 38: 236-242, 2016.

14. Franceschelli S, Rosati A, Lerose R, De Nicola S, Turco MC and Pascale M: Bag3 gene expression is regulated by heat shock factor 1. J Cell Physiol 215: 575-577, 2008.

15. Colvin TA, Gabai VL, Gong J, Calderwood SK, Li H, Gummuluru S, Matchuk ON, Smirnova SG, Orlova NV, Zamulaeva IA, et al: Hsp70-Bag3 interactions regulate cancer-related signaling networks. Cancer Res 74: 4731-4740, 2014.

16. Staibano S, Mascolo M, Di Benedetto M, Vecchione ML, Ilardi G, Di Lorenzo G, Autorino R, Salerno V, Morena A, Rocco A, et al: BAG3 protein delocalisation in prostate carcinoma. Tumour Biol 31: 461-469, 2010.

17. Tang JT, Wang JL, Du W, Hong J, Zhao SL, Wang YC, Xiong H, Chen HM and Fang JY: MicroRNA 345, a methylation-sensitive microRNA is involved in cell proliferation and invasion in human colorectal cancer. Carcinogenesis 32: 1207-1215, 2011.

18. Ammirante M, Rosati A, Arra C, Basile A, Falco A, Festa M, Pascale M, d'Avenia M, Marzullo L, Belisario MA, et al: IKK gamma\} protein is a target of BAG3 regulatory activity in human tumor growth. Proc Natl Acad Sci USA 107: 7497-7502, 2010.

19. Yunoki T, Tabuchi Y, Kondo T, Ishii Y and Hayashi A: Overexpression of the anti-apoptotic protein BAG3 in human choroidal melanoma: A case report. Oncol Lett 13: 4169-4172, 2017.

20. Boiani M, Daniel C, Liu X, Hogarty MD and Marnett LJ: The stress protein BAG3 stabilizes Mcl-1 protein and promotes survival of cancer cells and resistance to antagonist ABT-737. J Biol Chem 288: 6980-6990, 2013.

21. Chen Y, Yang LN, Cheng L, Tu S, Guo SJ, Le HY, Xiong Q, Mo R, $\mathrm{Li} \mathrm{CY}$, Jeong JS, et al: Bcl2-associated athanogene 3 interactome analysis reveals a new role in modulating proteasome activity. Mol Cell Proteomics 12: 2804-2819, 2013.

22. Ito T, Hayashida M, Kobayashi S, Muto N, Hayashi A, Yoshimura $\mathrm{T}$ and Mori $\mathrm{H}$ : Serine racemase is involved in d-aspartate biosynthesis. J Biochem 160: 345-353, 2016.

23. Furusawa Y, Yamanouchi Y, Iizumi T, Zhao WL, Mitsuhashi Y, Morita A, Enomoto A, Tabuchi Y and Kondo T: Checkpoint kinase 2 is dispensable for regulation of the p53 response but is required for $\mathrm{G} 2 / \mathrm{M}$ arrest and cell survival in cells with p53 defects under heat stress. Apoptosis 22: 1225-1234, 2017.

24. Watson JV, Chambers SH and Smith PJ: A pragmatic approach to the analysis of DNA histograms with a definable G1 peak. Cytometry 8: 1-8, 1987.

25. Hijikata A, Kitamura H, Kimura Y, Yokoyama R, Aiba Y, Bao Y, Fujita S, Hase K, Hori S, Ishii Y, et al: Construction of an open-access database that integrates cross-reference information from the transcriptome and proteome of immune cells. Bioinformatics 23: 2934-2941, 2007.

26. Makino S, Fukumura R and Gondo Y: Illegitimate translation causes unexpected gene expression from on-target out-of-frame alleles created by CRISPR-Cas9. Sci Rep 6: 39608, 2016. 
27. Kwan JA, Schulze CJ, Wang W, Leon H, Sariahmetoglu M, Sung M, Sawicka J, Sims DE, Sawicki G and Schulz R: Matrix metalloproteinase-2 (MMP-2) is present in the nucleus of cardiac myocytes and is capable of cleaving poly (ADP-ribose) polymerase (PARP) in vitro. FASEB J 18: 690-692, 2004.

28. Aldonyte R, Brantly M, Block E, Patel J and Zhang J: Nuclear localization of active matrix metalloproteinase-2 in cigarette smoke-exposed apoptotic endothelial cells. Exp Lung Res 35: $59-75,2009$.

29. McDermott U, Ames RY, Iafrate AJ, Maheswaran S, Stubbs H, Greninger P, McCutcheon K, Milano R, Tam A, Lee DY, et al: Ligand-dependent platelet-derived growth factor receptor (PDGFR)-alpha activation sensitizes rare lung cancer and sarcoma cells to PDGFR kinase inhibitors. Cancer Res 69: 3937-3946, 2009.

30. Tang Z, Arjunan P, Lee C, Li Y, Kumar A, Hou X, Wang B, Wardega P, Zhang F, Dong L, et al: Survival effect of PDGF-CC rescues neurons from apoptosis in both brain and retina by regulating GSK3beta phosphorylation. J Exp Med 207: 867-880, 2010.

31. Lucero CM, Vega OA, Osorio MM, Tapia JC, Antonelli M, Stein GS, van Wijnen AJ and Galindo MA: The cancer-related transcription factor Runx 2 modulates cell proliferation in human osteosarcoma cell lines. J Cell Physiol 228: 714-723, 2013.

32. Sugimoto H, Nakamura M, Yoda H, Hiraoka K, Shinohara K, Sang M, Fujiwara K, Shimozato O, Nagase H and Ozaki T: Silencing of RUNX2 enhances gemcitabine sensitivity of p53-deficient human pancreatic cancer AsPC-1 cells through the stimulation of TAp63-mediated cell death. Cell Death Dis 6: e1914, 2015.

33. Krishnan A, Nair SA and Pillai MR: Biology of PPAR gamma in cancer: A critical review on existing lacunae. Curr Mol Med 7 : 532-540, 2007.

34. Owens TW, Rogers RL, Best S, Ledger A, Mooney AM, Ferguson A, Shore P, Swarbrick A, Ormandy CJ, Simpson PT, et al: Runx2 is a novel regulator of mammary epithelial cell fate in development and breast cancer. Cancer Res 74: 5277-5286, 2014.

35. Sharma C, Pradeep A, Pestell RG and Rana B: Peroxisome proliferator-activated receptor gamma activation modulates cyclin D1 transcription via beta-catenin-independent and cAMP-response element-binding protein-dependent pathways in mouse hepatocytes. J Biol Chem 279: 16927-16938, 2004.

36. Shimura T, Ochiai Y, Noma N, Oikawa T, Sano Y and Fukumoto M: Cyclin D1 overexpression perturbs DNA replication and induces replication-associated DNA double-strand breaks in acquired radioresistant cells. Cell Cycle 12: 773-782, 2013.

37. Wang G, Woods A, Sabari S, Pagnotta L, Stanton LA and Beier F: RhoA/ROCK signaling suppresses hypertrophic chondrocyte differentiation. J Biol Chem 279: 13205-13214, 2004.
38. Parrales A, Palma-Nicolás JP, López E and López-Colomé AM Thrombin stimulates RPE cell proliferation by promoting c-Fos-mediated cyclin D1 expression. J Cell Physiol 222: 302-312, 2010

39. Benini S, Perbal B, Zambelli D, Colombo MP, Manara MC, Serra M, Parenza M, Martinez V, Picci P and Scotlandi K: In Ewing's sarcoma CCN3(NOV) inhibits proliferation while promoting migration and invasion of the same cell type. Oncogene 24: 4349-4361, 2005.

40. Mendoza-Naranjo A, El-Naggar A, Wai DH, Mistry P, Lazic N, Ayala FR, da Cunha IW, Rodriguez-Viciana P, Cheng H, Tavares Guerreiro Fregnani JH, et al: ERBB4 confers metastatic capacity in Ewing sarcoma. EMBO Mol Med 5: 1087-1102, 2013.

41. Capone C, Dabertrand F, Baron-Menguy C, Chalaris A, Ghezali L, Domenga-Denier V, Schmidt S, Huneau C, Rose-John S, Nelson MT and Joutel A: Mechanistic insights into a TIMP3-sensitive pathway constitutively engaged in the regulation of cerebral hemodynamics. Elife 5: pii: e17536, 2016.

42. Zins K, Pomyje J,Hofer E, Abraham D, Lucas T and Aharinejad S Egr-1 upregulates Siva-1 expression and induces cardiac fibroblast apoptosis. Int J Mol Sci 15: 1538-1553, 2014.

43. Baron V, De Gregorio G, Krones-Herzig A, Virolle T, Calogero A, Urcis R and Mercola D: Inhibition of Egr-1 expression reverses transformation of prostate cancer cells in vitro and in vivo. Oncogene 22: 4194-4204, 2003.

44. Jackson HW, Defamie V, Waterhouse P and Khokha R: TIMPs: Versatile extracellular regulators in cancer. Nat Rev Cancer 17: 38-53, 2017.

45. Tien YT, Chang MH, Chu PY, Lin CS, Liu CH and Liao AT: Downregulation of the KLF4 transcription factor inhibits the proliferation and migration of canine mammary tumor cells. Vet J 205: 244-253, 2015.

46. Liu YN, Abou-Kheir W, Yin JJ, Fang L, Hynes P, Casey O, Hu D, Wan Y, Seng V, Sheppard-Tillman H, et al: Critical and reciprocal regulation of KLF4 and SLUG in transforming growth factor $\beta$-initiated prostate cancer epithelial-mesenchymal transition. Mol Cell Biol 32: 941-953, 2012.

47. Kroepil F, Dulian A, Vallböhmer D, Geddert H, Krieg A, Vay C, Topp SA, am Esch JS, Baldus SE, Gires O, et al: High EpCAM expression is linked to proliferation and lauren classification in gastric cancer. BMC Res Notes 6: 253, 2013.

48. Liu Z, Shen J, Pu K, Katus HA, Plöger F, Tiefenbacher CP, Chen X and Braun T: GDF5 and BMP2 inhibit apoptosis via activation of BMPR2 and subsequent stabilization of XIAP. Biochim Biophys Acta 1793: 1819-1827, 2009.

49. Li Q, Kannan A, Das A, Demayo FJ, Hornsby PJ, Young SL, Taylor RN, Bagchi MK and Bagchi IC: WNT4 acts downstream of BMP2 and functions via $\beta$-catenin signaling pathway to regulate human endometrial stromal cell differentiation. Endocrinology 154: 446-457, 2013. 